\title{
Sensitive RHEED signature of Ti-excess enabling enhanced cationic composition control during the molecular beam epitaxy of $\mathrm{SrTiO}_{3}$ based solid solutions
}

\author{
Masoumeh Razaghi, Marc d'Esperonnat, Claude Botella, Sébastien Cueff, Romain Bachelet and \\ Guillaume Saint-Girons
}

Institut des Nanotechnologies de Lyon, INL-CNRS/UMR5270, Ecole Centrale de Lyon, 36 avenue Guy de Collongue 69134 Ecully, France

\begin{abstract}
Molecular beam epitaxy (MBE) is the best suited technique to engineer perovskite oxide properties, thanks to individual atom evaporation from elemental sources. Unfortunately, significant source drift often prevents exploiting this advantage, and improving the control of the composition of MBE grown oxide thin layers remains a challenge. In this context, in-situ reflection high energy electron diffraction (RHEED) has been for long identified as a useful tool, as surface reconstructions and RHEED oscillations depend on oxide cationic composition. We show here that monitoring the appearance of half-order streaks along the [210] RHEED azimuths of Ti-rich surfaces provides enhanced control of the cationic composition of $\mathrm{SrTiO}_{3}$ thin layers as compared to the more common strategy relying on [100] azimuths monitoring. We also provide quantitative evaluation of the uncertainty on composition control enabled by this method, namely $+/-6.7 \%$. In the end, we describe an original procedure to control the composition of perovskite oxide quaternary solid solutions.
\end{abstract}




\section{INTRODUCTION}

Functional oxides with perovskite structure (general formula $\mathrm{ABO}_{3}$ ) present a wide range of physical properties (conductivity, ferromagnetism, ferroelectricity, piezoelectricity, thermoelectricity,...) attractive in various domains. ${ }^{1}$ These properties depend on composition, and in particular on the ratio $[A] /[\mathrm{B}]$ between the cation concentrations, ${ }^{2,3}$ which must be accurately controlled. $\mathrm{SrTiO}_{3}$ (STO), archetypal perovskite oxide, is used in non-volatile memories, smart sensors, energy harvesters,.... Bulk stoichiometric STO $([\mathrm{A}] /[\mathrm{B}]=1)$ is a band insulator. It becomes ferroelectric with tunable Curie temperature when submitted to partial isovalent cationic substitution of Ba on A site. ${ }^{4}$ Partial aliovalient substitution (typically of La on A site or $\mathrm{Nb}$ on B site) ${ }^{5,6}$ or introduction of oxygen vacancies ${ }^{7}$ in STO induces tunable electrical conductivity (with high carrier mobility) and remarkable thermoelectric properties. When subjected to cationic stoichiometry deviation $([A] /[B] \neq 1)$, STO shows resistive switching behavior, ${ }^{8}$ enhanced charge carrier mobility, ${ }^{9}$ as well as tunable electrical and thermal conductivity. ${ }^{10,11}$

Molecular beam epitaxy (MBE) is particularly well suited to control perovskite oxide composition and physical properties thanks to elemental sources. ${ }^{12}$ However, such control remains to date a challenge due to source drift. The latter causes poor growth reproducibility, which makes inoperable calibration strategies using post-growth (ex-situ) composition measurements (based, for instance, on X-ray diffraction (XRD)). Monitoring Reflection High Energy Electron Diffraction (RHEED) oscillations enables in-situ STO composition control with an accuracy in the $1 \%$ range. ${ }^{13}$ However, this procedure is not strictly speaking real-time as it requires employing $\mathrm{SrO} / \mathrm{TiO}_{2}$ monolayers $(\mathrm{ML})$ alternated growth and observing oscillations during a large number of alternations, which is not compatible with the growth of any structure. Consequently, as ex-situ calibrations although to a lesser extent, its efficiency is limited by source drift. Solving the source drift issue requires a truly real-time procedure, which is the case of STO(001) surface reconstructions monitoring using in-situ RHEED. ${ }^{14,15,16}$ This method, the accuracy of which has to date not been reported, relies on the dependence of these reconstructions on the $\mathrm{Sr} / \mathrm{Ti}$ surface coverage. ${ }^{17,18,19,20,21}$ It is operated by monitoring the appearance of half-order streaks along the [100] (respectively [110]) RHEED azimuth, signature Ti-rich (respectively Sr-rich) surfaces, and by tuning the elemental growth rates to prevent their formation, ${ }^{14,15}$ or to maintain a $\mathrm{TiO}_{2}$-saturated STO surface in the case of metalorganic assisted oxide $\mathrm{MBE}^{16}$ where titanium tetraisopropoxide (TTIP) used as Ti source enables self-limited Ti incorporation. ${ }^{22,23}$

In this context, we show here that the observation of half-order streaks along both [100] and [210] azimuths associated with the Ti-rich STO surface reconstruction is preceded by that of half-order streaks along the [210] azimuth only, and that using this signature enables significant accuracy 
improvement for in-situ real-time STO composition control. We quantify this accuracy, and compare it to that provided by high resolution X-ray diffraction measurements (XRD). In the end, we propose a strategy to control the cationic composition of quaternary $(\mathrm{La}, \mathrm{Sr}) \mathrm{TiO}_{3}$ solid solutions using RHEED reconstructions.

\section{EXPERIMENTAL}

The samples considered in the following were grown by solid source MBE on (001)-oriented STO substrates under a molecular oxygen partial pressure of $5 \times 10^{-7}$ Torr at $700^{\circ} \mathrm{C}$, as detailed in the supplementary material.

In a first experiment, one $\mathrm{SrO} \mathrm{ML}$ was deposited on a clean, $\mathrm{TiO}_{2}$-terminated STO substrate treated using a HF-based buffered oxide etchant (BOE), ${ }^{24}$ followed by one $\mathrm{TiO}_{2} \mathrm{ML}$ to recover the initial complete $\mathrm{TiO}_{2}$ termination. During this process, the RHEED pattern along the [110] azimuth was continuously recorded. The complete operation was reproduced twice in order to record the evolution of the RHEED patterns along the [100] and [210] azimuths.

In a second experiment, a series of $\sim 30 \mathrm{~nm}$ thick STO epitaxial layers with various cationic compositions was grown. X-ray photoemission spectroscopy (XPS) was used to estimate the $[\mathrm{Sr}] /[\mathrm{Ti}]$ atomic concentrations ratio, with a relative uncertainty in the $\pm 2-3 \%$ range. ${ }^{25}$ XRD (respectively X-ray reflectivity (XRR)) was used to measure the out-of-plane lattice parameter c (respectively the thickness) of these layers.

In the end, in a third experiment, three $\mathrm{La}_{x} \mathrm{Sr}_{1-\mathrm{x}} \mathrm{TiO}_{3}$ (LSTO) / STO heterostructures with $\mathrm{x} \sim 0.2$ and $[\mathrm{A}] /[\mathrm{B}]=([\mathrm{La}]+[\mathrm{Sr}]) / \mathrm{Ti} \sim 1$ were grown. Spectroscopic ellipsometry was used to evaluate the charge carrier concentration and hence the La substitution ratio $\mathrm{x}$.

\section{RESULTS AND DISCUSSION}

As mentioned earlier, STO surface reconstructions depend on $\mathrm{Sr} / \mathrm{Ti}$ surface coverage in usual MBE conditions. $50 \% \mathrm{SrO} / 50 \% \mathrm{TiO}_{2} \mathrm{STO}(001)$ surfaces are reconstruction-free, ${ }^{14}$ Ti-rich surfaces present two-domain $(2 \times 1), c(6 \times 2), c(4 \times 4)$ or $c(4 \times 2)$ reconstructions depending on oxygen partial pressure, ${ }^{17,18,19,26}$ and Sr-rich surfaces present a $\sqrt{2} \times \sqrt{2} R 45^{\circ}$ reconstruction. ${ }^{21}$ The results of the first experiment (alternated $\mathrm{SrO} / \mathrm{TiO}_{2}$ deposition), summarized in Fig.1, are consistent with this well documented behavior. 
(a)
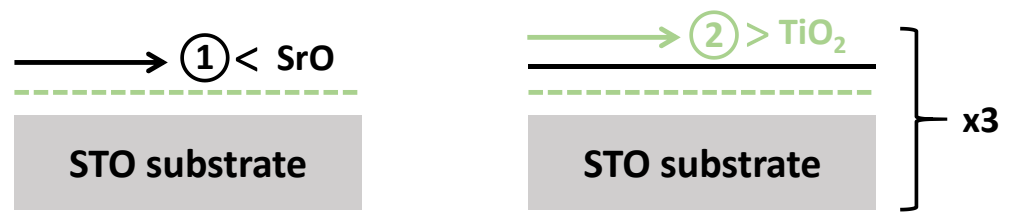

(b)

$\mathrm{TiO}_{2}$ coverage

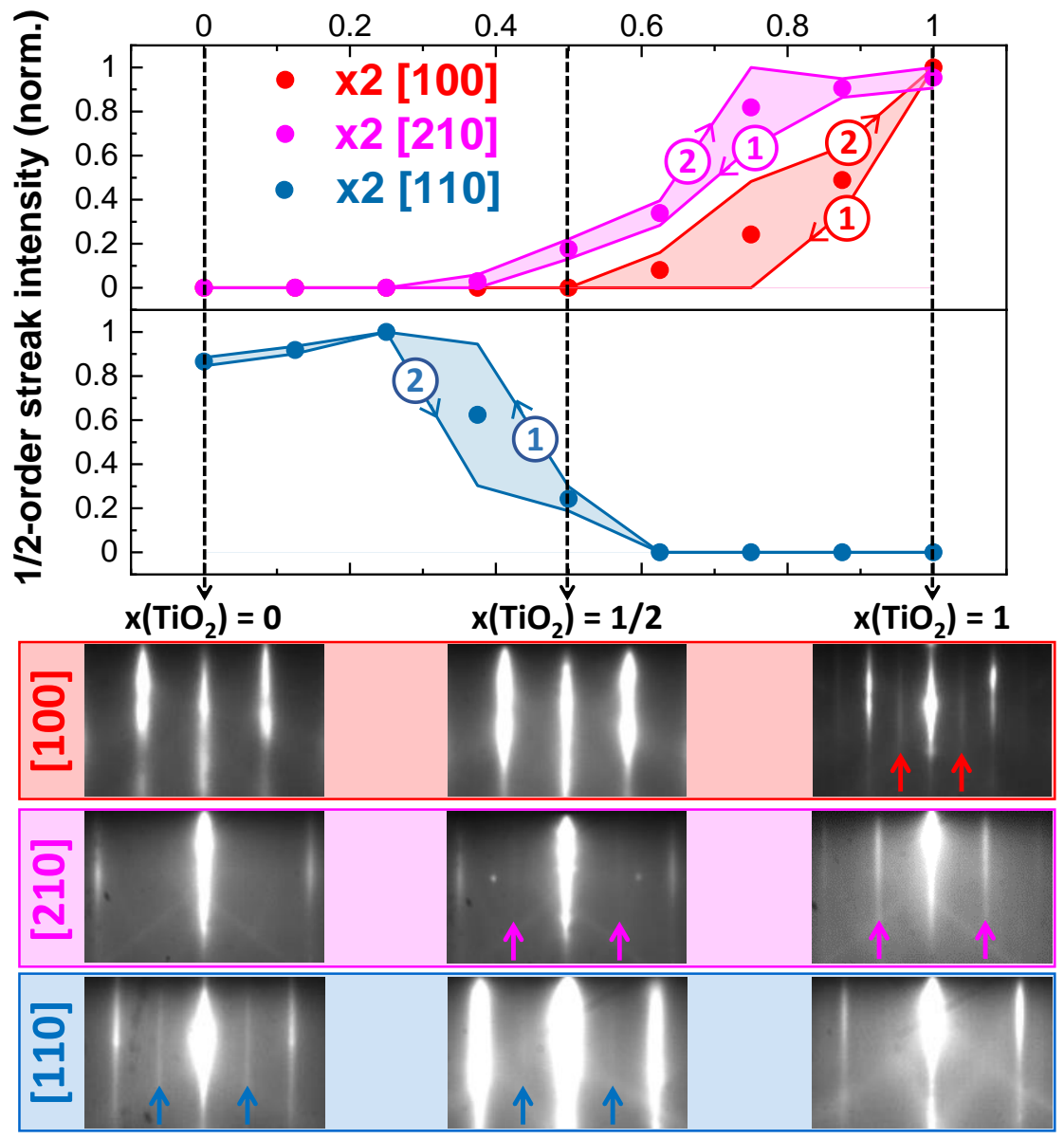

Fig.1 : (a) Growth sequence used during the first experiment. On a $\mathrm{TiO}_{2}$ terminated $\mathrm{STO}(001)$ substrate $\left(---=\right.$ initial complete $\mathrm{TiO}_{2}$ termination), a SrO ML is deposited ( $\longrightarrow$ (1) $<$ ) until complete SrO coverage is reached (_) while monitoring the evolution of the RHEED pattern along one azimuth. Then, a $\mathrm{TiO}_{2} \mathrm{ML}$ is deposited ( $\longrightarrow$ (2)> ), keeping on with RHEED monitoring along the same azimuth. This procedure is carried out three times to record the RHEED pattern evolution along the [110], [100] and [210] azimuths. (b) Evolution of the intensity of the half-order streaks along the [100], [210] and [110] azimuths during SrO deposition (arrowheads labelled 1 on curves) and $\mathrm{TiO}_{2}$ deposition (arrowheads labelled 2 on curves) as a function of the $\mathrm{TiO}_{2}$ coverage $\times\left(\mathrm{TiO}_{2}\right)$ (the $\mathrm{SrO}$ coverage $\times(\mathrm{SrO})$ obeys $\left.x(\mathrm{SrO})=1-x\left(\mathrm{TiO}_{2}\right)\right)$. Bottom panel: RHEED patterns recorded at different $\mathrm{TiO}_{2}$ coverages. Arrows highlight the half-order streaks. 
The RHEED pattern evolves with surface coverage from half-order streaks along the [110] azimuth for a complete SrO coverage $\left(x\left(\mathrm{TiO}_{2}\right)=0\left(\sqrt{2} \times \sqrt{2} R 45^{\circ} \text {-reconstructed Sr-rich surface }\right)^{21}\right.$ to half-order streaks along both [100] and [210] azimuths for a complete $\mathrm{TiO}_{2}$ coverage $\left(x\left(\mathrm{TiO}_{2}\right)=1,(2 \times 1)\right.$ reconstructed Ti-rich surface ${ }^{17,27}$ ). Notwithstanding a slight hysteresis, this evolution does not strongly depend on whether $\mathrm{SrO}$ or $\mathrm{TiO}_{2}$ is the topmost (growing) atomic layer.

On the Sr-rich side, the intensity of the half-order streaks along the [110] azimuth becomes maximal for a SrO coverage of $\left.\sim 0.75 \mathrm{ML}\left(\mathrm{x}\left(\mathrm{TiO}_{2}\right) \sim 0.25\right)\right)$, consistent with Ref.21 stipulating that the $\sqrt{2} \times$ $\sqrt{2} R 45^{\circ}$ reconstruction is a partially SrO-deficient SrO-terminated surface. On the Ti-rich side, the intensity of the half-order streaks along the [100] azimuth is maximal for $x\left(\mathrm{TiO}_{2}\right)=1$, as expected from some models of the two-domain $(2 \times 1)$-reconstructed Ti-rich surface involving periodic arrangement of $\mathrm{O}$ vacancies in a complete $\mathrm{TiO}_{2}$ surface atomic plane. ${ }^{17,28}$

Interestingly, the half-order streaks along the [210] azimuth, sometimes associated with another signature of the two-domain (2x1)-reconstruction, ${ }^{14}$ appear at lower $\mathrm{TiO}_{2}$ coverage than the half-order streaks along the [100] azimuth and their intensity is maximum at lower $\mathrm{TiO}_{2}$ coverage too (namely $\left.x\left(\mathrm{TiO}_{2}\right) \sim 0.8\right)$. One may speculate that atomic ordering at the origin of the $(2 x 1)$ reconstruction is anisotropic, starting earlier along $<210>$ surface axes, or that the formation of the $(2 \times 1)$ reconstruction is preceded by another surface atomic arrangement leading to half-order streaks along the [210] azimuths only. Either way, this shows that half-order streaks along the [210] azimuths are an earlier signature of Ti-excess on the STO surface than half-order streaks along the [100] azimuths, conventionaly used as marker to monitor STO composition during growth. ${ }^{14}$

Lastly, for $x\left(\mathrm{TiO}_{2}\right)=1 / 2$, half-order streaks are detected along both [210] and [110] azimuths, suggesting the coexistence of reconstructed $\mathrm{SrO}$-terminated and $\mathrm{TiO}_{2}$-terminated domains in equal proportion. Noticeably, in codeposition conditions ( $\mathrm{Sr}$ and Ti deposited simultaneously), half-order streaks are absent from the RHEED patterns over a significant composition range around $x\left(\mathrm{TiO}_{2}\right)=1 / 2$, probably due to larger surface disorder and larger growth rate.

We now show that tracking the half-order streaks along the [210] azimuth enables improved STO composition control. The out-of-plane lattice parameters of the STO layers of the second experiment (measured by XRD) are shown as a function of their cationic composition (measured by XPS and indexed by $\delta$ in $\mathrm{Sr}_{1+\delta} \mathrm{TiO}_{3}$ ) in Fig.2, along with the RHEED patterns observed at the end of the growth. 


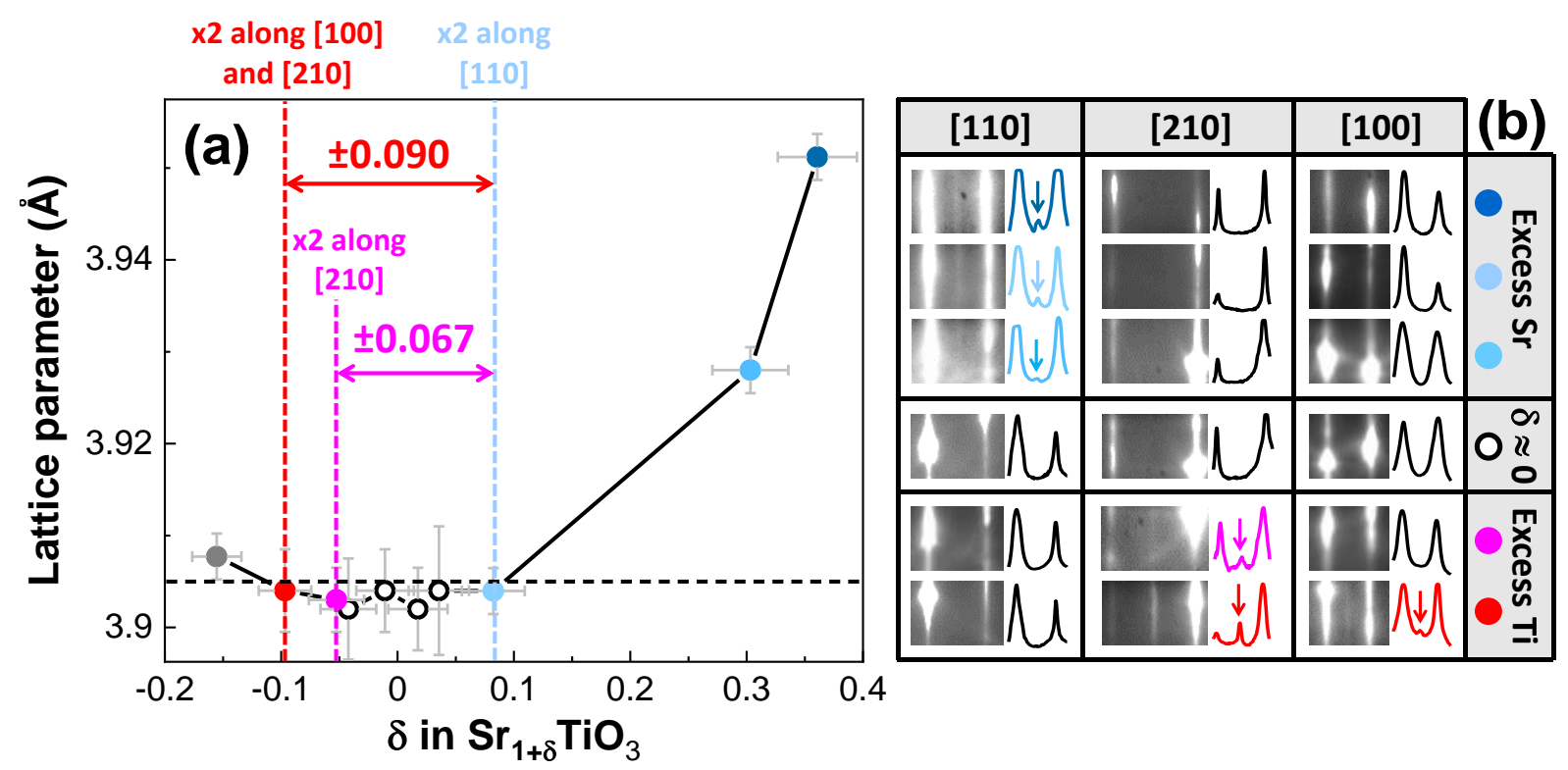

Fig.2 : (a) Out-of-plane lattice parameter of the STO layers as a function of their cationic composition indexed by $\delta \mathrm{in} \mathrm{Sr}_{1+\delta} \mathrm{TiO}_{3}$. The dot color code corresponds to the surface reconstruction observed at the end of the growth : $\bullet, \bullet, \diamond=x 2$ along the [110] azimuth, $\mathbf{O}=$ no reconstruction $(\delta \approx 0), \bullet=x 2$ along [210] only, $\bullet=x 2$ along both [210] and [100], and $\bigcirc=$ no reconstruction on the very Ti-rich surface due to surface disorder. -----.. indicate the value of bulk STO lattice parameter (3.905 A). Zooms on the corresponding RHEED patterns (selected region is shown in the supplementary material) are displayed in (b), along with intensity profiles. Arrows highlight the half-order streaks.

As expected, the STO lattice parameter is minimal and close to the bulk value when $\delta$ is near 0 , and increases for larger $|\delta|$ values as defects due to deviation from stoichiometric composition cause lattice unit-cell inflation. ${ }^{29,11,30,26}$ The composition range over which the lattice parameter remains constant and minimal is quite large, corresponding to $\delta= \pm 9 \%$. Deviation from stoichiometric composition goes hand in hand with the observation of the RHEED patterns commented above, as excess cations segregate toward the surface or accumulate at the growth front. On the Sr-rich side, half-order streaks along the [110] azimuth are detected when $\delta$ exceeds 0.08 , while on the Ti-rich side, half-order streaks along both [100] and [210] azimuths are detected when $\delta$ gets smaller than -0.09 . Besides, for $\delta=-$ 0.16 , surface reconstructions are no longer observed due to increased surface disorder and roughness caused by Ti excess. The strategy of STO composition control based on monitoring the half-order streaks along the [110] and [100] azimuths ${ }^{14,15}$ thus provides an uncertainty of about $\pm 9 \%$ on $\delta$ estimation, comparable to that provided by ex-situ XRD measurements. As for sub-ML $\mathrm{TiO}_{2}$ and $\mathrm{SrO}$ alternated growth, half-order streaks along the [210] azimuths provide an earlier signature of Ti excess on the surface than half-order streaks along the [100] azimuths, and allow detecting smaller Ti excesses in the layers, corresponding to $\delta<-0.05$. This shows that monitoring the appearance of half-order 
streaks along the [110] and [210] azimuths (instead of [110] and [100]) significantly improves the accuracy on STO composition control, reducing the uncertainty on $\delta$ down to $\pm 6.7 \%$, which is also better than that provided by XRD measurements. Such improvement is particularly valuable in the context detailed in the introduction where STO properties are strongly affected by deviations from the stoichiometric $[\mathrm{Sr}] /[\mathrm{Ti}]$ ratio.

As mentioned in the introduction, partially substituting divalent $\mathrm{Sr}$ by trivalent $\mathrm{La}$ in $\mathrm{SrTiO}_{3}$ to form $\left(\mathrm{La}_{x}, \mathrm{Sr}_{1-\mathrm{x}}\right) \mathrm{TiO}_{3}(\mathrm{LSTO})$ allows obtaining N-type thin layers, the electrical conductivity and thermoelectric properties of which can be tuned by varying the La concentration. ${ }^{31,32,33,34}$ We present in the following a method to control in-situ the composition of such LSTO thin layers using RHEED reconstructions.

The three LSTO/STO heterostructures considered for these purpose were grown as follows (third experiment): the $10 \mathrm{~nm}$ thick STO layer was grown at constant $\mathrm{Sr}$ beam equivalent pressure $\mathrm{F}_{0}(\mathrm{Sr})$ measured before growth using a Bayard-Alpert gauge. During STO growth, the Ti cell temperature was tuned to prevent the appearance of half-order streaks along the [110] and [210] azimuths of the RHEED pattern, so that the resulting STO layer has stoichiometric composition within $\pm 6.7 \%$ uncertainty. Practically speaking, this was done by repeating the following sequence:

(i) constant monitoring of the [110] and [210] RHEED azimuths during Sr and Ti codeposition

(ii) closing of the Sr (respectively Ti) shutter each time half-order streaks are observed along the [110] (respectively [210]) azimuth until their complete disappearance (to compensate the surface cationic excess)

(iii) growth stop and increase (respectively decrease) of the Ti cell temperature to approach stoichiometric growth conditions

(iv) growth re-start

until half order streaks are no longer observed along any azimuth.

Then, for the growth of the LSTO layers with a targeted La atomic composition of $20 \%$ (( $\left.\left.\mathrm{La}_{0.2} \mathrm{Sr}_{0.8}\right) \mathrm{TiO}_{3}\right)$, the Ti cell temperature was kept constant to the value leading to stoichiometric STO growth, and the $\mathrm{Sr}$ cell temperature was reduced so that the $\mathrm{Sr}$ beam equivalent pressure is $0.8 \times F_{0}(S r)$. LSTO growth was then started by co-depositing $\mathrm{La}, \mathrm{Sr}$ and $\mathrm{Ti}$, and the La cell temperature was adjusted to obtain three different RHEED patterns for each of the three samples, namely half-order streaks along the [110] azimuth for one sample, no surface reconstruction for the second sample, and half-order streaks along both [100] and [210] azimuths, using the 4-step procedure described above. 
Fig. 3 shows the RHEED patterns (recorded at the end of the growth) and the XRD radial scans measured on these heterostructures.

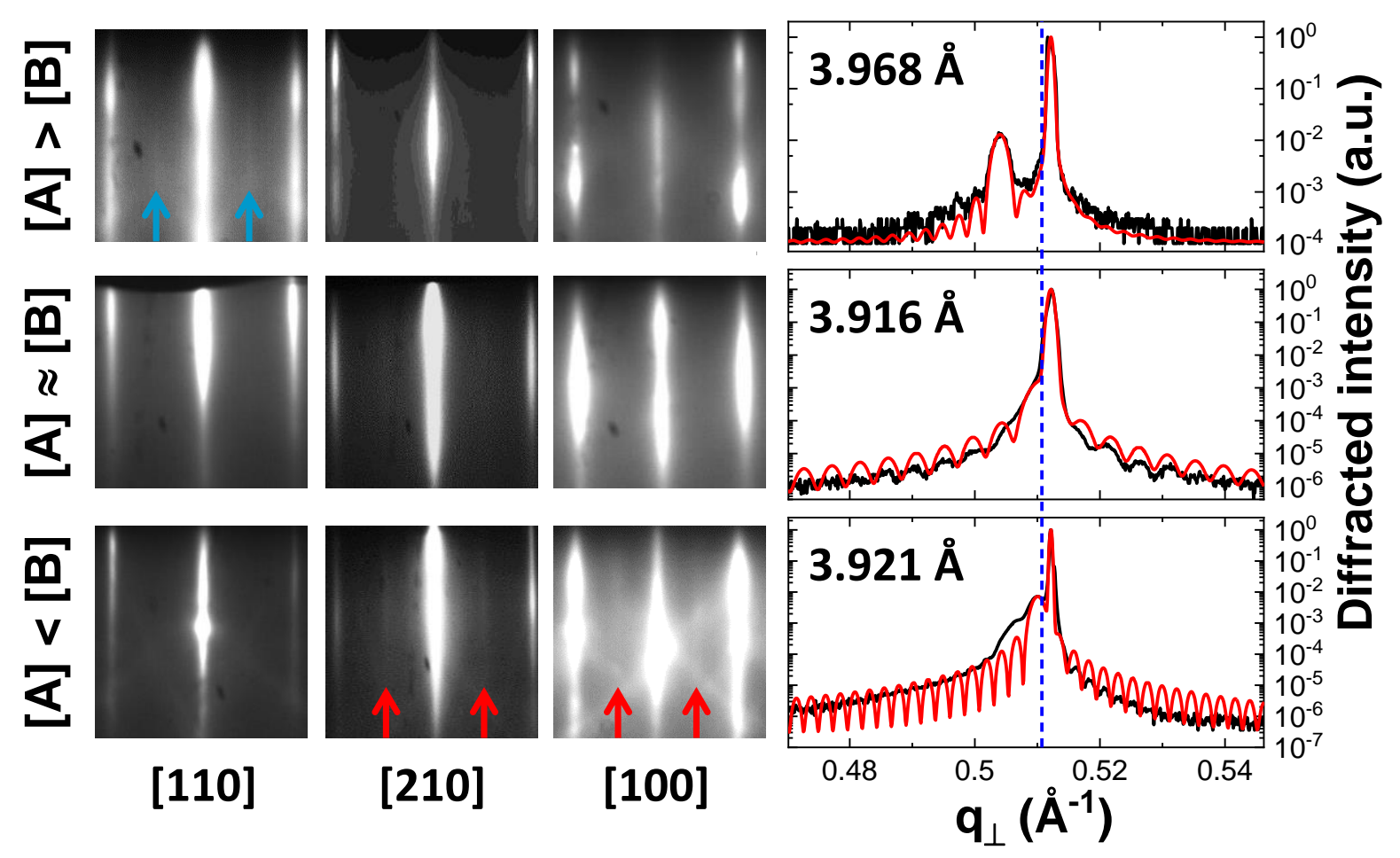

Fig.3 :RHEED patterns (at the end of the growth, half-order streaks highlighted by arrows) and XRD radial scans recorded on LSTO/STO heterostructures with various compositions $(-=$ experimental curves, $\_$fit). $[A]$ (respectively [B]) designates the total A site (respectively B site) composition in $\mathrm{ABO}_{3} . q_{\perp}$ stands for the out-of-plane diffraction vector. indicate the position of the maximum of the nearly-stoichiometric LSTO diffraction peak (corresponding to a lattice parameter of $3.916 \AA$ ).

For the sample presenting a reconstruction-free RHEED pattern at the end of LSTO growth $([A] \approx[B]$ in Fig.3), the LSTO lattice parameter (3.916 $\AA$ ) is minimal. For the sample presenting half-order streaks along the [110] RHEED azimuth ([A] > [B] in Fig.3), as for that presenting half-order streaks along both [210] and $[100]$ azimuths $([A]<[B]$ in Fig.3), the lattice parameter is increased, to $3.968 \AA$ and $3.921 \AA$, respectively. This shows that LSTO behaves as STO with respect to cationic composition in terms of lattice parameter inflation and surface reconstructions, and that therefore the latter can be used to monitor composition in-situ using RHEED. It has to be noted here that as in the case of STO, half-order streaks appear at lower surface Ti excess along the [210] azimuth than along the [100] azimuth (not shown).

We now discuss the properties of the LSTO/STO heterostructure grown in near stoichiometric conditions $([A] \approx[B]$ in Fig.3), depicted in Fig.4(a). 

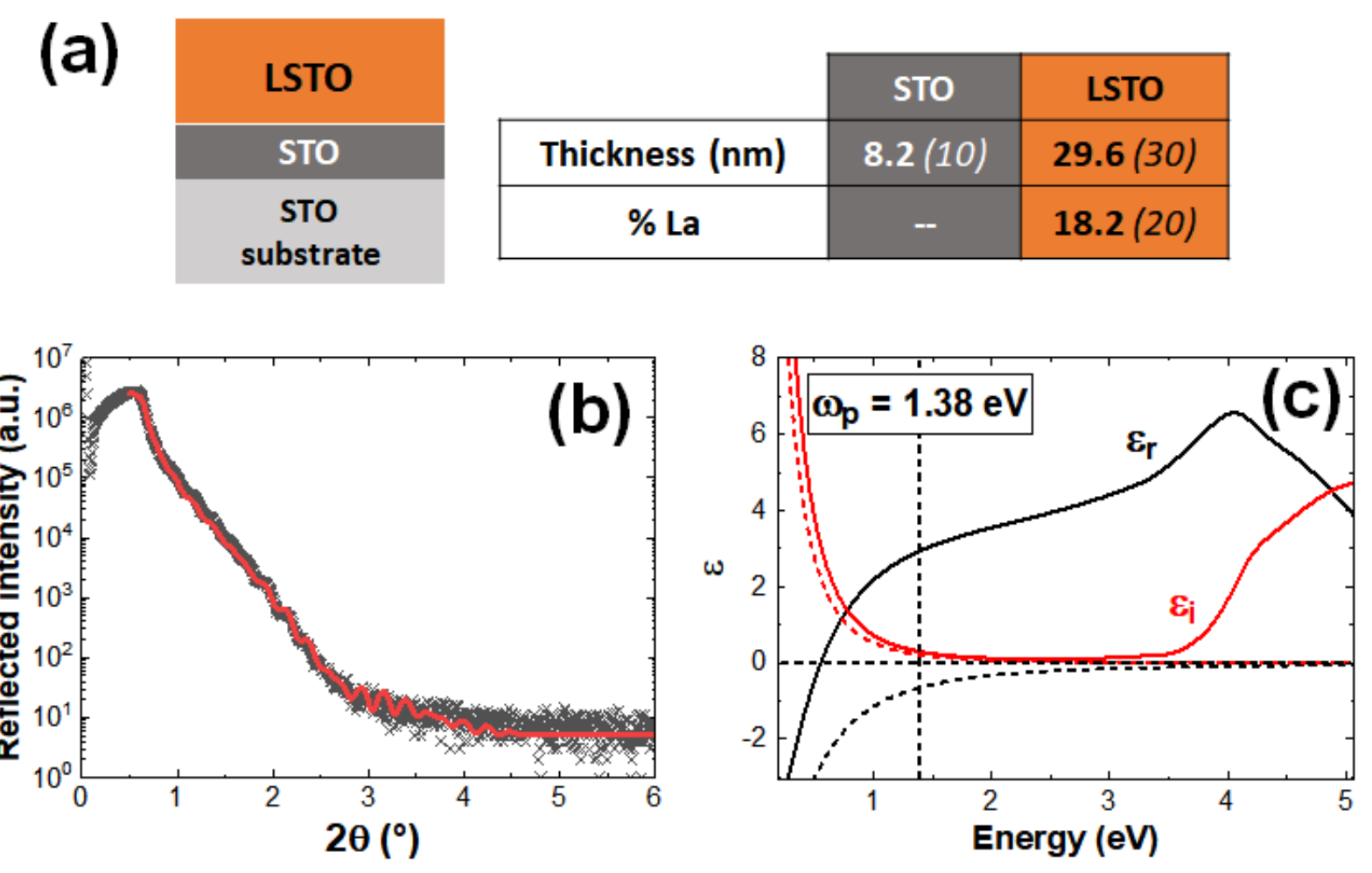

Fig.4: (a) Schematic of the LSTO/STO structure grown in near stoichiometric conditions, and associated thicknesses and La composition respectively deduced from XRR and spectrometric ellipsometry measurements. In the table, measured values are bolded and nominal (targeted) values are bracketed. (b) XRR spectrum recorded on the sample (_) and associated fit (_). (c) Real ( $\varepsilon_{r}, \ldots$ ) and imaginary ( $\varepsilon_{i}$ __ ) parts of the permittivity of the STO layer measured by spectroscopic ellipsometry. The free charge carrier contribution in the low energy range can be described by a Drude contribution (----,----).

The XRD radial scan displayed in Fig.3 attests for the excellent structural quality of the LSTO layer, with an excellent fit between the experimental data and the model calculated for an ideal structure, free of any defect. The lattice parameter of the LSTO layer, namely $3.916 \pm 10^{-3} \AA$, is slightly larger than the 3.907-3.908 $\AA$ expected from theoretical calculations for bulk $\left(\mathrm{La}_{0.2} \mathrm{Sr}_{0.8}\right) \mathrm{TiO}_{3},{ }^{35,36}$ but is among the lowest reported experimental values, $37,38,39,40$ suggesting successful control of the layer composition and a low concentration of defects prone to inflate the unit cell volume. The thicknesses of the STO and LSTO layers estimated from XRR are reasonably close from the targeted values (table in Fig.4), which is another indication of the reliability of the method. The permittivity of the LSTO layer was measured using spectroscopic ellipsometry (Fig.4(c)). It presents a free charge carrier contribution with an increase of the imaginary part $\varepsilon_{\mathrm{i}}$ and a decrease of its real part $\varepsilon_{\mathrm{r}}$ below $\sim 1.5 \mathrm{eV}$. This behavior is usually described by a Drude function $\varepsilon_{D}{ }^{41}$ reading: 
$\varepsilon_{D}(\omega)=-\frac{\omega_{p}^{2}}{-\omega^{2}+i \cdot \Gamma_{D} \omega}$

where $\omega$ is the frequency, $\Gamma_{\mathrm{D}}$ a broadening term and $\omega_{\mathrm{p}}$ the plasma frequency, related to the free charge carrier concentration $\mathrm{N}$ by :

$$
\omega_{p}=\sqrt{\frac{N \cdot e^{2}}{\varepsilon_{0} \cdot m^{*}}}
$$

where e is the electron charge, $\varepsilon_{0}$ the vacuum permittivity and $\mathrm{m}^{*}$ the charge carrier effective mass. Excellent fit of the ellipsometry data was obtained with $\omega_{\mathrm{p}}=1.38 \mathrm{eV}\left(2.094 \times 10^{15} \mathrm{rad} . \mathrm{s}^{-1}\right.$, see supplementary material). The free electron mass in LSTO is about $2.2 \times \mathrm{m}_{0},{ }^{42,57}$ where $\mathrm{m}_{0}=9.11 \times 10^{-31}$ $\mathrm{kg}$ is the electronic mass, leading to a free electron concentration $\mathrm{N}$ of about $3.03 \times 10^{21} \mathrm{~cm}^{-3}$ (Eq.2). As every La atom delivers one free electron to the structure, ${ }^{51}$ this corresponds to a La atomic concentration of about $18.2 \%$, for a unit-cell volume of $59.71 \AA^{3}\left(=3.905^{2} \times 3.916 \AA^{3}\right)$. This La atomic concentration is very close to the targeted $20 \%$ value, further confirming the efficiency of our optimized RHEED based procedure to control the composition of complex perovskite oxide solid solutions.

\section{CONCLUSION}

The study reported here shows that half-order streaks along the [210] RHEED azimuths are an earlier signature of Ti-excess on the STO surface than half-order streaks along the [100] azimuths, and that this can be leveraged to improve the accuracy in controlling STO composition in-situ and in real-time using RHEED monitoring. This work also estimates the corresponding uncertainty on composition control, namely $+/-6.7 \%$ using the [210] azimuth, to be compared to +/- $9 \%$ using the [100] azimuth. Moreover, we describe a procedure to control the composition of perovskite oxide quaternary solid solutions. These results pave the way towards an improved control of the properties of $\mathrm{SrTiO}_{3}$ based solid solutions, which strongly depend on their composition. Furthermore, the method can be operated in-situ and in real-time during growth, enabling dynamic correction of elemental cell drift. It does not impose constraints on the growth conditions, and in particular it does not require using layerby-layer growth, in contrast to RHEED oscillation based methods..$^{13}$ Besides, accuracy on composition control can be improved by reducing the growth rate, which should reduce surface roughness and thus allow detecting half-order streaks at lower Sr or Ti excesses. The method can also be automated, using feedback loops to control effusion cell temperatures from half-order streak intensities. In the end, the method can be applied to any perovskite oxide for which composition-dependent surface 
reconstructions are observed, which is at least the case for STO, for $\left(\mathrm{La}_{x} \mathrm{Sr}_{1-x}\right) \mathrm{TiO}_{3}$ solid solutions as shown here, for ferroelectric $\left(\mathrm{Ba}_{\mathrm{x}}, \mathrm{Sr}_{1-\mathrm{x}}\right) \mathrm{TiO}_{3}$ alloys ${ }^{43}$ and probably many others.

\section{SUPPLEMENTARY MATERIAL}

See the supplementary material for the complete RHEED data, the method used to estimate the thin layer composition using XPS, the complete X-ray diffraction data, the detailed procedure used for STO and LSTO growth, and full details on ellipsometry measurements.

\section{ACKNOWLEDGMENTS}

The authors gratefully thank Philippe Regreny and Jean-Baptiste Goure for their technical support. This work benefited from the support of the projects LILIT ANR-16-CE24-0022, MITO ANR-17-CE05-0018 and DIAMWAFEL ANR-15-CE08-0034.

\section{REFERENCES}

1 L.J. Tejuca, and J.L.G. Fierro, "Properties and Applications of Perovskite Type Oxides", Eds Marcel Dekker, New York (1993).

2 C.N.R. Rao, J. Gopalakrishnan and K. Vidyasagar, Indian J. Chem. Sect. A 23A, 265 (1984).

3 D.M. Smyth, Annu. Rev. Mater. Sci. 15, 329 (1985).

4 A.D. Hilton, and B.W. Ricketts, J. Phys. D: Appl. Phys. 29, 1321 (1996).

5 T. Okuda, K. Nakanishi, S. Miyasaka, and Y. Tokura, Phys. Rev. B 63, 113104 (2001).

6 J. Son, P. Moetakef, B. Jalan, O. Bierwagen, N.J. Wright, R. Engel-Herbert, and S. Stemmer, Nature Materials 9, 482 (2010).

7 H.N. Lee, S.S.A. Seo, W.S. Choi, and C.M. Rouleau, Scientific Reports 6, 19941 (2016).

8 E. Mikheev, J. Hwang, A.P. Kajdos, A.J. Hauser, and S. Stemmer, Scientific Reports 5, 11079 (2015).

9 S. Kobayashi, Y. Mizumukai, T. Ohnishi, N. Shibata, Y. Ikuhara, and T. Yamamoto, ACS Nano 9, 10769 (2015).

10 T. Ohnishi, K. Shibuya, T. Yamamoto, and M. Lippmaa, J. Appl. Phys. 103, 103703 (2008).

11 C.M. Brooks, R.B. Wilson, A. Schäfer, J.A. Mundy, M.E. Holtz, D.A. Muller, J. Schubert, D.G. Cahill, and D.G. Schlom, Appl. Phys. Lett. 107, 051902 (2015). 
12 D.G. Schlom, L.Q. Chen, X. Pan, A. Schmehl, and M.A. Zurbuchen, J. Am. Ceram. Soc. 91, 2429, (2008).

13 J.H. Haeni, C.D. Theis, and D.G. Schlom, Journal of Electroceramics 4, 385, (2000).

14 Z. Yu, Y. Liang, C. Overgaard, X. Hu, J. Curless, H. Li, Y. Wei, B. Craigo, D. Jordan, R. Droopad, J. Finder, K. Eisenbeiser, D. Marshall, K. Moore, J. Kulik, and P. Fejes, Thin Solid Films 462, 51, (2004).

15 P. Fisher, H. Du, M. Skowronski, P.A. Salvador, O. Maksimov, and X. Weng, J. Appl. Phys. 103, 013519, (2008).

16 A.P. Kajdos, and S. Stemmer, Appl. Phys. Lett. 105, 191901, (2014).

17 M.R. Castell, Surf Sci 505, 1 (2002).

18 N. Erdman, K.R. Poeppelmeier, M. Asta, O. Warschkow, D.E. Ellis, and L.D. Marks, Nature 419, 56, (2002).

19 O. Warschkow, M. Asta, N. Erdman, K.R. Poeppelmeier, D.E.Ellis, L.D.Marks, Surf Sci 573, 446 (2004). 20 Q.D. Jiang, and J. Zegenhagen, Surf. Sci. 425, 343 (1999).

21 S. Ogawa, K. Kato, N. Nagatsuka, S. Ogura, and K. Fukutani, Phys. Rev. B 96, 085303 (2017).

22 B. Jalan, R. Engel-Herbert, N.J. Wright, and S. Stemmer, J. Vac. Sci. Technol. A 27, 461 (2009).

23 B. Jalan, P. Moetakef, and S. Stemmer, Appl. Phys. Lett. 95, 032906 (2009).

24 G. Koster, B.L. Kropman, G.J.H.M. Rijnders, D.H.A. Blank, and H. Rogalla, Appl. Phys. Lett. 73, 2920, (1998).

25 R. Hesse, T. Chassé, P. Streubel and R. Szargan, Surf. Interf. Anal. 36, 1373, (2004).

26 Q.D. Jiang, and J.Zegenhagen, Surf. Sci. 425, 343 (1999).

27 N. Erdman, O. Warschkow, M. Asta, K. R. Poeppelmeier, D. E. Ellis, and L. D. Marks, J. Am. Chem. Soc. 125,10050 (2003).

28 N. Erdman, O. Warschkow, M. Asta, K. R. Poeppelmeier, D. E. Ellis, and L. D. Marks, J. Am. Chem. Soc. 125,10050 (2003).

29 C.M. Brooks, L. Fitting Kourkoutis, T. Heeg, J. Schubert, D. A. Muller, and D. G. Schlom, Appl. Phys. Lett. 94, 162905 (2009).

30 T. Ohnishi, K. Shibuya, T. Yamamoto, and M. Lippmaa, J. Appl. Phys. 103, 103703 (2008).

31 T. Okuda, K. Nakanishi, S. Miyasaka, and Y. Tokura, Phys. Rev. B 63, 113104 (2001).

32 B. Jalan, and S. Stemmer, Appl Phys Lett. 97, 42106 (2010).

33 J. Son, P. Moetakef, B. Jalan, O. Bierwagen, N.J. Wright, R. Engel-Herbert, and S. Stemmer, Nature Materials 9, 482 (2010).

34 J. Ravichandran, W. Siemons, H. Heijmerikx, M. Huijben, A. Majumdar, and R. Ramesh, Chem Mater.

22, 3983 (2010).

35 R. Moos, and K.H. Härdtl, J. Am. Ceram. Soc. 80, 2549 (1997). 
36 A. Janotti, B. Jalan, S. Stemmer, and C.G. Van de Walle, Appl. Phys. Lett. 100, 262104 (2012).

37 J. Ravichandran, W. Siemons, M. L. Scullin, S. Mukerjee, M. Huijben, J. E. Moore, A. Majumdar, and R. Ramesh, Phys. Rev. B 83, 035101 (2011).

38 W.S. Choi, H.K. Yoo, and H. Ohta, Adv. Funct. Mater. 25, 799 (2015).

39 M. Choi, A.B. Posadas, C.A. Rodriguez, A. O'Hara, H. Seinige, A.J. Kellock, M.M. Frank, M. Tsoi, S. Zollner, V. Narayanan, and A.A. Demkov, J. Appl. Phys. 116, 043705 (2014).

40 M. Apreutesei, R. Debord, M. Bouras, P. Regreny, C. Botella, A. Benamrouche, A. CarreteroGenevrier, J. Gazquez, G. Grenet, S. Pailhès, G Saint-Girons, and R. Bachelet, Sci. Technol. Adv. Mater. 18, 435 (2017).

41 W.L. Bade, J. Chem. Phys. 27, 1280 (1957).

42 M. Bouras, D. Han, S. Cueff, R. Bachelet, and G. Saint-Girons, ACS Photonics 6, 1755 (2019).

43 Y. Yoneda, K. Sakaue and H. Terauchi, Surf. Sci. 529, 283 (2003). 